\title{
Stochastic stability for Markovian jump linear systems associated with a finite number of jump times ${ }^{\text {th }}$
}

\author{
João B.R. do Val, ${ }^{\mathrm{a}, *}$ Cristiane Nespoli, ${ }^{\mathrm{b}}$ and Yusef R.Z. Cáceres ${ }^{\mathrm{a}}$ \\ ${ }^{a}$ UNICAMP_Univ. Est. de Campinas, Fac. de Eng. Elétrica, Depto. de Telemática, C.P. 6101, \\ 13081-970 Campinas, SP, Brazil \\ b UNESP_Univ. Est. Paulista, Fac. de Ciências e Tecnologia, Depto. de Matemática, C.P. 1234 , \\ 19060-400 Pres. Prudente, SP, Brazil \\ Received 6 March 2003 \\ Submitted by C.T. Leondes
}

\begin{abstract}
This paper deals with a stochastic stability concept for discrete-time Markovian jump linear systems. The random jump parameter is associated to changes between the system operation modes due to failures or repairs, which can be well described by an underlying finite-state Markov chain. In the model studied, a fixed number of failures or repairs is allowed, after which, the system is brought to a halt for maintenance or for replacement. The usual concepts of stochastic stability are related to pure infinite horizon problems, and are not appropriate in this scenario. A new stability concept is introduced, named stochastic $\tau$-stability that is tailored to the present setting. Necessary and sufficient conditions to ensure the stochastic $\tau$-stability are provided, and the almost sure stability concept associated with this class of processes is also addressed. The paper also develops equivalences among second order concepts that parallels the results for infinite horizon problems.

(c) 2003 Elsevier Inc. All rights reserved.
\end{abstract}

Keywords: Markov jump linear systems; Stochastic stability; Maintenance model

\footnotetext{
Research supported in part by CNPq, Grant 300721/86-2 and the IM-AGIMB project, and by the PRONEX Grant 015/98 'Control of Dynamical Systems.' A preliminary version was presented at the 2002 American Control Conference, Anchorage.

* Corresponding author

E-mail address: jbosco@dt.fee.unicamp.br (J.B.R.do Val).
}

0022-247X/\$ - see front matter ㄷ 2003 Elsevier Inc. All rights reserved. doi:10.1016/S0022-247X(03)00424-4 


\section{Introduction}

Let us consider a discrete-time linear system subject to abrupt parameter changes that can be modelled via a discrete-time finite-state Markov chain. Here, these changes are associated with failures or repairs of the running system. This class of systems is known in the literature as discrete-time Markovian jump linear systems (MJLS); see (1) and (2).

The study of MJLS, and in particular, the study of stochastic stability of MJLS has attracted the attention of many researchers. Ji and Chizeck [1] provided necessary and sufficient conditions to ensure second moment stochastic stability of MJLS. Ji et al. [2] established the equivalence among various second moment stochastic stability concepts, and the relation of these with almost sure stability. Costa and Fragoso [3], using Kronecker product, obtained necessary and sufficient conditions for mean square stability of these systems with additive noise included. Based on a stochastic version of the Lyapunov's second method, Fang [4] presented sufficient conditions for the almost sure stability of MJLS. Some less conservative conditions were derived by Li et al. [5].

Although, several stochastic stability concepts can be found in the literature, for the class of systems treated here those stochastic stability concepts are not adequate. A situation of interest arises when one studies this class of systems until the occurrence of a random event $\tau$, more specifically, a stopping time $\tau$ of the joint process $\left\{x_{k}, \theta_{k} ; k \geqslant 0\right\}$ described by (1) and (2). The stopping time $\tau$ may represent interesting situations from the point of view of applications. For instance, $\tau$ can be the accumulated $n$th failure and repair of the system. In an other situation, $\tau$ can represent the occurrence of a "crucial failure," (which may occur after a random number of failures). In both situations the system will be paralyzed after this event and the future behaviour is of no concern.

In this scenario, the usual stochastic stability concepts are not adequate, since they are suitable for pure infinite time horizon problems, but not for problems with unbounded but finite horizon defined by a stopping time as in the present setting. In this paper we propose a new stochastic stability concept associated with a class of stopping times of the joint process $\left\{x_{k}, \theta_{k} ; k \geqslant 0\right\}$ for MJLS, called stochastic $\tau$-stability. It is appropriate to deal with the problem with horizon connected to the accumulated $n$th failure or repair of the system, and it will be shown that every stochastic stable system is $\tau$-stable, but the converse is not true.

The paper is organized as follows. In Section 2 the basic definitions are presented, in Section 3 the necessary and sufficient conditions for $\tau$-stability are derived and referred to the stochastic stability concept by means of an example. In Section 4 equivalences among concepts of $\tau$-stability are presented, and in Section 5 the concept of $\tau$ almost sure stability is considered.

\section{Notation and problem formulation}

Throughout this paper, the following notation is adopted. $\mathbb{R}^{n}$ denotes the $n$-dimensional real space and $\mathcal{M}^{m \times n}\left(\mathcal{M}^{m}\right)$ is the normed linear space of all $m \times n(m \times m)$ real matrices. The transpose of matrix $U$ is indicated by $U^{\prime}$ and a nonnegative definite matrix (positive definite) is represented by $U \geqslant 0(U>0)$. Thus, the closed convex (opened) of all the non- 
negative definite (positive definite) matrices in $\mathcal{M}^{m}$ is denoted by $\mathcal{M}^{m 0}=\left\{U \in \mathcal{M}^{m}: U=\right.$ $\left.U^{\prime} \geqslant 0\right\}\left(\mathcal{M}^{m+}\right)$. The linear space of all sequences of $s$ real matrices in $\mathcal{M}^{m \times n}\left(\mathcal{M}^{m}\right)$ is represented by $\mathbb{M}^{m \times n}=\left\{\mathbf{U}=\left(U_{1}, \ldots, U_{s}\right): U_{i} \in \mathcal{M}^{m \times n}, i \in\{1, \ldots, s\}\right\}\left(\mathbb{M}^{m}\right)$. For the sake of notational simplification, $\mathbb{M}^{m 0}$ is written when $U_{i} \in \mathcal{M}^{m 0}$, for all $i \in\{1, \ldots, s\}$ and $\mathbb{M}^{m+}$ is written when $U_{i} \in \mathcal{M}^{m+}$. The standard vector norm in $\mathbb{R}^{n}$ is indicated by $\|\cdot\|$ and the corresponding induced norm of matrix $U$ by $\|U\|$. In addition, $\lambda(U)$ indicates an eigenvalue and $r_{\sigma}(U)$ the spectral radius of $U \in \mathcal{M}^{m}$, respectively. Let $\mathbb{1}_{\{.\}}$be the Dirac measure. For $\mathbf{U} \in \mathbb{M}^{m 0}$, the following operator is defined:

$$
\mathcal{E}_{i}(\mathbf{U})=\sum_{j \neq i} p_{i j} U_{j}
$$

Consider the discrete-time Markovian jump linear systems (MJLS) defined on the fundamental probability space $\left(\Omega, \mathfrak{F},\left\{\mathfrak{F}_{k}\right\}, P\right)$,

$$
\mathcal{S}: \quad x_{k+1}=A_{\theta_{k}} x_{k}
$$

where $\left\{x_{k}, \theta_{k} ; k \geqslant 0\right\}$ are the states of process with values in $\mathbb{R}^{r} \times \mathfrak{X} ;\left\{\theta_{k} ; k \geqslant 0\right\}$ is a time homogeneous Markov chain taking values in a finite set $\mathfrak{X}=\{1, \ldots, E\}$, with initial distribution $\mu$ and transition probability matrix $\mathbb{P}=\left[p_{i j}\right]$, where

$$
p_{i j}:=P\left(\theta_{k+1}=j \mid \theta_{k}=i\right), \quad \forall i, j \in \mathfrak{X}, k \geqslant 0 .
$$

The set $\mathfrak{X}$ comprises the various operation modes of the system (1) and for each $\theta_{k}=$ $i \in \mathfrak{X}$, the matrix $A_{\theta_{k}} \in \mathcal{M}^{r}$ (associated to " $i$ th" mode), will be assigned as $A_{\theta_{k}}:=A_{i}$. We mention that $A_{\theta_{k}}$ may encompasses jump-dependent linear controls of type $G_{\theta_{k}} x_{k}$. The MJLS as defined is trivially a strong Markov process.

This class of stochastic systems is associated to systems subject to failures in their components or connections according to a Markov chain. The situation that we are interested in arises when one wishes to study the stability of such a system until the occurrence of a fixed number $N$ of failures and repairs. The paper recognizes the sequence of $\left\{\mathcal{F}_{k}\right\}$ stopping times containing the successive times of the occurrence of such failures and then it studies the stability of systems (1) and (2) according to these stopping times. Let us introduce first the concepts of second moment $\tau$-stochastic stability.

Definition 1. Consider a $\left\{\mathfrak{F}_{k}\right\}$-stopping time $\tau$. The MJLS $\mathcal{S}$ is

(i) Stochastically $\tau$-stable ( $\tau$-SS) if for each initial condition $x_{0}$ and initial distribution $\mu$

$$
E\left[\sum_{k \geqslant 0}\left\|x_{k}\right\|^{2} \mathbb{1}_{\{\tau \geqslant k\}}\right]<\infty
$$

(ii) Mean square $\tau$-stable ( $\tau$-MSS) if for each initial condition $x_{0}$ and initial distribution $\mu$

$$
E\left[\left\|x_{k}\right\|^{2} \mathbb{1}_{\{\tau \geqslant k\}}\right] \rightarrow 0 \quad \text { when } k \rightarrow \infty
$$

(iii) Exponentially mean square $\tau$-stable ( $\tau$-EMSS) if there exist constants $0<\alpha<1$ and $\beta>0$, such that for each initial condition $x_{0}$ and initial distribution $\mu$

$$
E\left[\left\|x_{k}\right\|^{2} \mathbb{1}_{\{\tau \geqslant k\}}\right] \leqslant \beta \alpha^{k}\left\|x_{0}\right\|^{2}, \quad k \geqslant 0 .
$$




\section{Stochastic $\tau$-stability}

In this section, necessary and sufficient conditions are given for a certain class of $\left\{\mathfrak{F}_{k}\right\}$ stopping times. Let us define the sequence $\mathcal{T}^{N}=\left\{T_{n} ; n=0,1, \ldots, N\right\}$ of stopping times associated to jump times:

$$
\begin{aligned}
& T_{0}=0, \\
& T_{n}=\min \left\{k>T_{n-1}: \theta_{k} \neq \theta_{T_{n-1}}\right\}, \quad n=1,2, \ldots, N ;
\end{aligned}
$$

eventually, $T_{n}=\cdots=T_{N}=+\infty$ for some $n \geqslant 1$, with probability one. In this work, we assume that $\tau=T_{n}$ for some finite $n$. The next lemma, which the proof is omitted, will be used as support. For notational simplicity we sometimes write $E_{k}[\cdot]$ instead of $E\left[\cdot \mid x_{k}, \theta_{k}\right]$.

Lemma 2. For all $m \geqslant 1$ and $i, j \in \mathfrak{X}$

$$
P\left(T_{1}=m, \theta_{m}=j \mid \theta_{0}=i\right)= \begin{cases}p_{i j} \mathbb{1}_{\{m=1\}} & \text { if } p_{i i}=0, \\ p_{i i}{ }^{m-1} p_{i j} \mathbb{1}_{\left\{p_{i i}<1\right\}} & \text { if } p_{i i}>0 .\end{cases}
$$

Note that $P\left(T_{1}=1 \mid \theta_{0}=i\right)=1$ and $P\left(T_{1}=+\infty \mid \theta_{0}=i\right)=1$, whenever $p_{i i}=0$ and $p_{i i}=1$, respectively.

Theorem 3. For $\tau \in \mathcal{T}^{N}$, the following assertions are equivalent:

(i) The MJLS $\mathcal{S}$ is $\tau$-SS.

(ii) For any given set of matrices $\mathbf{Q} \in \mathbb{M}^{r+}$, there exists a unique set of matrices $\mathbf{L} \in \mathbb{M}^{n+}$, satisfying the Lyapunov equations

$$
p_{i i} A_{i}^{\prime} L_{i} A_{i}-L_{i}+Q_{i}=0, \quad \forall i \in \mathfrak{X} .
$$

(iii) $r_{\sigma}\left(p_{i i}^{1 / 2} A_{i}\right)<1$, for all $i \in \mathfrak{X}$.

Proof. (ii) $\Leftrightarrow$ (iii) This equivalence is well known from the Lyapunov stability theory, see [6].

(i) $\Leftarrow$ (ii) The proof employs an induction argument on the sequence $\mathcal{T}^{N}$. First, define the functional

$$
V_{k}(x, i):=x^{\prime}\left(P_{i} \mathbb{1}_{\left\{T_{1}>k\right\}}+S_{i} \mathbb{1}_{\left\{T_{1}=k\right\}}\right) x,
$$

where $S_{i} \in \mathcal{M}^{r+}$, and $P_{i} \in \mathcal{M}^{r+}$ is the solution of

$$
p_{i i} A_{i}^{\prime} P_{i} A_{i}-P_{i}+Q_{i}+A_{i}^{\prime} \mathcal{E}_{i}(\mathbf{S}) A_{i}=0, \quad \forall i \in \mathfrak{X} .
$$

The existence of such $\mathbf{P} \in \mathbb{M}^{r+}$ relies on (ii). Hence, we can write that

$$
\begin{aligned}
& E_{k}\left[V_{k+1}\left(x_{k+1}, \theta_{k+1}\right)-V_{k}\left(x_{k}, \theta_{k}\right)\right] \\
& =-x_{k}^{\prime} S_{\theta_{k}} x_{k} \mathbb{1}_{\left\{T_{1}=k\right\}}+E_{k}\left[\left(x_{k+1}^{\prime} S_{\theta_{k+1}} x_{k+1}-x_{k} P_{\theta_{k}} x_{k}\right) \mathbb{1}_{\left\{T_{1}=k+1\right\}}\right] \\
& \quad+E_{k}\left[\left(x_{k+1}^{\prime} P_{\theta_{k+1}} x_{k+1}-x_{k} P_{\theta_{k}} x_{k}\right) \mathbb{1}_{\left\{T_{1}>k+1\right\}}\right] .
\end{aligned}
$$

Calculating the expected values above, we get that 


$$
\begin{aligned}
& E_{k}\left[V_{k+1}\left(x_{k+1}, \theta_{k+1}\right)-V_{k}\left(x_{k}, \theta_{k}\right)\right] \\
& \quad=-x_{k}^{\prime} S_{\theta_{k}} x_{k} \mathbb{1}_{\left\{T_{1}=k\right\}}+x_{k}^{\prime}\left[p_{\theta_{k} \theta_{k}} A_{\theta_{k}}^{\prime} P_{\theta_{k}} A_{\theta_{k}}+A_{\theta_{k}}^{\prime} \mathcal{E}_{\theta_{k}}(\mathbf{S}) A_{\theta_{k}}-P_{\theta_{k}}\right] x_{k} \mathbb{1}_{\left\{T_{1}>k\right\}},
\end{aligned}
$$

since $P\left(T_{1}=k+1 \mid \theta_{k}\right)=1-p_{\theta_{k} \theta_{k}}$ and $P\left(T_{1}>k+1 \mid \theta_{k}\right)=p_{\theta_{k} \theta_{k}}$.

However, if there exists $\mathbf{L} \in \mathbb{M}^{r+}$ solving (6) then there is $\mathbf{P} \in \mathbb{M}^{r+}$ solving (7), and the previous relation can be written as

$$
E_{k}\left[V_{k+1}\left(x_{k+1}, \theta_{k+1}\right)-V_{k}\left(x_{k}, \theta_{k}\right)\right]=-x_{k}^{\prime}\left(Q_{\theta_{k}} \mathbb{1}_{\left\{T_{1}>k\right\}}+S_{\theta_{k}} \mathbb{1}_{\left\{T_{1}=k\right\}}\right) x_{k} .
$$

Now, observe that

$$
\begin{aligned}
& \sum_{k=0}^{\kappa} E_{0}\left[V_{k+1}\left(x_{k+1}, \theta_{k+1}\right)-V_{k}\left(x_{k}, \theta_{k}\right)\right] \\
& \quad=\sum_{k=0}^{\kappa} E_{0}\left[E_{k}\left[V_{k+1}\left(x_{k+1}, \theta_{k+1}\right)-V_{k}\left(x_{k}, \theta_{k}\right)\right]\right] .
\end{aligned}
$$

Applying (8), and considering that $\mathbf{Q}, \mathbf{S} \in \mathbb{M}^{r+}$,

$$
\begin{aligned}
& E_{0}\left[V_{\kappa+1}\left(x_{\kappa+1}, \theta_{\kappa+1}\right)\right]-V_{0}\left(x_{0}, \theta_{0}\right) \\
& =-\sum_{k=0}^{\kappa} E_{0}\left[x_{k}^{\prime}\left(Q_{\theta_{k}} \mathbb{1}_{\left\{T_{1}>k\right\}}+S_{\theta_{k}} \mathbb{1}_{\left\{T_{1}=k\right\}}\right) x_{k} \mathbb{1}_{\left\{T_{1} \geqslant k\right\}}\right] \\
& \quad \leqslant-\sum_{k=0}^{\kappa} \delta E_{0}\left[\left\|x_{k}\right\|^{2} \mathbb{1}_{\left\{T_{1} \geqslant k\right\}}\right]
\end{aligned}
$$

for some $\delta>0$. Since $E_{0}\left[V_{k}\left(x_{k}, \theta_{k}\right)\right] \geqslant 0, \forall k \geqslant 0$, we get from (9) that

$$
\limsup _{\kappa \rightarrow \infty} \sum_{k=0}^{\kappa} E_{0}\left[\left\|x_{k}\right\|^{2} \mathbb{1}_{\left\{T_{1} \geqslant k\right\}}\right] \leqslant \frac{1}{\delta} V_{0}\left(x_{0}, \theta_{0}\right)<\infty
$$

holds. Therefore, the MJLS $\mathcal{S}$ is $\tau$-SS.

Now, for the sake of an induction argument, let us assume that for some $n$ the inequality

$$
\limsup _{\kappa \rightarrow \infty} \sum_{k=0}^{\kappa} E\left[x_{k}^{\prime} Q_{\theta_{k}} x_{k} \mathbb{1}_{\left\{T_{n} \geqslant k\right\}}\right]<x_{0}^{\prime} \hat{P}_{\theta_{0}} x_{0}
$$

holds and thus, by setting $\mathbf{Q} \equiv \mathbf{I}, E\left[\left\|x_{T_{n}}\right\|^{2} \mathbb{1}_{\left\{T_{n} \geqslant k\right\}}\right]<\infty$. However,

$$
\begin{aligned}
& \limsup _{\kappa \rightarrow \infty} \sum_{k=0}^{\kappa} E\left[x_{k}^{\prime} Q_{\theta_{k}} x_{k} \mathbb{1}_{\left\{T_{n+1} \geqslant k\right\}}\right] \\
& \quad=\limsup _{\kappa \rightarrow \infty} E\left[\sum_{k=0}^{\kappa} x_{k}^{\prime} Q_{\theta_{k}} x_{k} \mathbb{1}_{\left\{T_{n}>k\right\}}+\sum_{k=T_{n}}^{\kappa} x_{k}^{\prime} Q_{\theta_{k}} x_{k} \mathbb{1}_{\left\{T_{n} \leqslant k \leqslant T_{n+1}\right\}}\right] .
\end{aligned}
$$

Notice that using the strong Markov property and the homogeneity property, the second term conditioned to the knowledge of $\left(x_{T_{n}}, \theta_{T_{n}}\right)$ can be written as 


$$
\begin{aligned}
& \limsup _{\kappa \rightarrow \infty} E\left[\sum_{k=T_{n}}^{\kappa} x_{k}^{\prime} Q_{\theta_{k}} x_{k} \mathbb{1}_{\left\{T_{n+1} \geqslant k\right\}} \mid x_{T_{n}}, \theta_{T_{n}}\right] \\
& \quad=\limsup _{\kappa \rightarrow \infty} E\left[\sum_{k=0}^{\kappa-T_{n}} x_{k}^{\prime} Q_{\theta_{k}} x_{k} \mathbb{1}_{\left\{k \leqslant T_{1}\right\}} \mid x_{0}=x_{T_{n}}, \theta_{0}=\theta_{T_{n}}\right]<x_{\theta_{T_{n}}}^{\prime} \hat{P}_{\theta_{T_{n}}} x_{\theta_{T_{n}}} .
\end{aligned}
$$

Thus, one concludes from (10) and (11) that

$$
\begin{aligned}
& \limsup _{\kappa \rightarrow \infty} \sum_{k=0}^{\kappa} E\left[x_{k}^{\prime} Q_{\theta_{k}} x_{k} \mathbb{1}_{\left\{T_{n+1} \geqslant k\right\}}\right] \leqslant \limsup _{\kappa \rightarrow \infty} \sum_{k=0}^{\kappa} E\left[x_{k}^{\prime} Q_{\theta_{k}} x_{k} \mathbb{1}_{\left\{T_{n}>k\right\}}+x_{T_{n}}^{\prime} \hat{P}_{\theta_{T_{n}}} x_{T_{n}}\right] \\
& \quad \leqslant \limsup _{\kappa \rightarrow \infty} \sum_{k=0}^{\kappa} E\left[x_{k}^{\prime} Q_{\theta_{k}} x_{k} \mathbb{1}_{\left\{T_{n} \geqslant k\right\}}+x_{T_{n}}^{\prime}\left(\hat{P}_{\theta_{T_{n}}}-Q_{\theta_{T_{n}}}\right) x_{T_{n}}\right]<2 x_{0}^{\prime} \hat{P}_{\theta_{0}} x_{0} .
\end{aligned}
$$

Hence,

$$
\limsup _{\kappa \rightarrow \infty} \sum_{k=0}^{\kappa} E\left[\left\|x_{k}\right\|^{2} \mathbb{1}_{\left\{T_{n} \geqslant k\right\}}\right]<\infty \quad \text { for any } T_{n} \in \mathcal{T}^{N},
$$

implying $\tau$-SS.

(i) $\Rightarrow$ (ii) As in the previous part define the functional

$$
x_{0}^{\prime} P_{\theta_{0}} x_{0}:=E_{0}\left[\sum_{k=0}^{\infty} x_{k}^{\prime} Q_{\theta_{k}} x_{k} \mathbb{1}_{\left\{T_{1}>k\right\}}+x_{T_{1}}^{\prime} S_{\theta_{T_{1}}} x_{T_{1}}\right],
$$

for all $\left(x_{0}, \theta_{0}\right) \in \mathbb{R}^{r} \times \mathfrak{X}$. The right-hand side of (12) can be expressed as

$$
E_{0}\left[x_{0}^{\prime} Q_{\theta_{0}} x_{0}+E_{T_{1}}\left[\left(\sum_{k=1}^{\infty} x_{k}^{\prime} Q_{\theta_{k}} x_{k} \mathbb{1}_{\left\{T_{1}>k\right\}}+x_{T_{1}}^{\prime} S_{\theta_{T_{1}}} x_{T_{1}}\right) \mathbb{1}_{\left\{T_{1} \geqslant 1\right\}}\right]\right] .
$$

In addition,

$$
\begin{aligned}
& E_{T_{1}}\left[\left(\sum_{k=1}^{\infty} x_{k}^{\prime} Q_{\theta_{k}} x_{k} \mathbb{1}_{\left\{T_{1}>k\right\}}+x_{T_{1}}^{\prime} S_{\theta_{T_{1}}} x_{T_{1}}\right) \mathbb{1}_{\left\{T_{1} \geqslant 1\right\}}\right] \\
& =E_{T_{1}}\left[\left(\sum_{k=1}^{\infty} x_{k}^{\prime} Q_{\theta_{k}} x_{k} \mathbb{1}_{\left\{T_{1}>k\right\}}+x_{T_{1}}^{\prime} S_{\theta_{T_{1}}} x_{T_{1}}\right) \mathbb{1}_{\left\{T_{1}>1\right\}}+x_{T_{1}}^{\prime} S_{\theta_{T_{1}}} x_{T_{1}} \mathbb{1}_{\{T=1\}}\right] .
\end{aligned}
$$

Thus, in view of the strong Markov property, applying homogeneity in (14) and introducing it in (13), we have that

$$
x_{0}^{\prime} P_{\theta_{0}} x_{0}=x_{0}^{\prime} Q_{\theta_{0}} x_{0}+E_{0}\left[x_{1}^{\prime} P_{\theta_{1}} x_{1} \mathbb{1}_{\left\{T_{1}>1\right\}}+x_{T_{1}}^{\prime} S_{\theta_{T_{1}}} x_{T_{1}} \mathbb{1}_{\left\{T_{1}=1\right\}}\right] .
$$

Since $x_{0}$ is arbitrary, (15) implies that

$$
p_{i i} A_{i}^{\prime} P_{i} A_{i}+A_{i}^{\prime} \mathcal{E}_{i}(S) A_{i}-P_{i}=-Q_{i},
$$

using the fact that $P\left(T_{1}=1 \mid \theta_{0}=i\right)=1-p_{i i}$ and $P\left(T_{1}>1 \mid \theta_{0}=i\right)=p_{i i}$. Thus, from the Lyapunov stability theory, the existence of the set $\mathbf{L} \in \mathbb{M}^{r+}$ satisfying (6) is guaranteed, completing the proof for $n=1$. 
Now, for the general case, we can write from (i) that

$$
E\left[\sum_{k=0}^{\infty} x_{k}^{\prime} Q_{\theta_{k}} x_{k} \mathbb{1}_{\left\{T_{N}>k\right\}}+x_{T_{N}}^{\prime} S_{\theta_{T_{N}}} x_{T_{N}}\right]<\infty
$$

which implies, from the strong Markov property, that

$$
E_{T_{n}}\left[\sum_{k=T_{n}}^{\infty} x_{k}^{\prime} Q_{\theta_{k}} x_{k} \mathbb{1}_{\left\{T_{n+1}>k\right\}}+x_{T_{n+1}}^{\prime} S_{\theta_{T_{n+1}}} x_{T_{n+1}}\right]<\infty,
$$

for $n=0,1, \ldots, N-1$. By applying the homogeneity property, it follows that (16) is equivalent to (12) with $x_{0}=x_{T_{n}}$ and $\theta_{0}=\theta_{T_{n}}$, and the existence of a set of matrices $\mathbf{L} \in$ $\mathbb{M}^{r+}$ satisfying (6) is assured, then completing the proof.

Although the stability of each operation mode is neither necessary nor sufficient for stochastic stability of systems (1), as pointed out by Ji and Chizeck [1] (see also [3]), the stability of the $p_{i i}^{1 / 2} A_{i}$ for each $i$ is a necessary condition to ensure that stability notion. Notice however that, for MJLS with the present stability notion, the equivalent condition (iii) of $\tau$-stability in Theorem 3 is less restrictive. In fact, for such systems it can be shown that stochastic stability, as established in [1], implies stochastic $\tau$-stability, but the converse is not generally true. This is illustrated in the next example.

Example 4. Consider a two form scalar MJLS $\mathcal{S}$ with $\left|p_{i i}^{1 / 2} a_{i}\right|<1$ for $i=1,2$. Consequently, the system is $\tau$-SS according to Theorem 3. If system $\mathcal{S}$ is also stochastically stable as defined by $\mathrm{Ji}$ and Chizeck [1], the solutions of recursive equations in $l_{i}^{\kappa}$, given by

$$
l_{i}^{\kappa+1}=p_{i i} a_{i}^{2} l_{i}^{\kappa}+\left(1-p_{i i}\right) a_{i}^{2} l_{j}^{\kappa}+q_{i}, \quad i, j=1,2, j \neq i,
$$

are such that $l_{i}=\lim _{\kappa \rightarrow \infty} l_{i}^{\kappa}$, and $\left(l_{1}, l_{2}\right)$ is the solution of a coupled Lyapunov equation. Writing the preceding equation in matrix form, the eigenvalues of the matrix of recursion can be obtained from the expression

$$
\operatorname{det}\left(s \mathbf{I}-\left[\begin{array}{cc}
p_{11} a_{1}^{2} & \left(1-p_{11}\right) a_{1}^{2} \\
\left(1-p_{22}\right) a_{2}^{2} & p_{22} a_{2}^{2}
\end{array}\right]\right)=0 .
$$

This is equivalent to

$$
s^{2}-\left(p_{11} a_{1}^{2}+p_{22} a_{2}^{2}\right) s+\left(a_{1} a_{2}\right)^{2}\left(p_{11}+p_{22}-1\right)=0 .
$$

Notice from (18) that, if the module of the eigenvalues of the recursive matrix are greater or equal than one, then

$$
\left|\left(a_{1} a_{2}\right)^{2}\left(p_{11}+p_{22}-1\right)\right| \geqslant 1 \text {. }
$$

On the other hand, given that $\left|p_{i i} a_{i}^{2}\right|<1$ for $i=1,2$, then

$$
\left(a_{1} a_{2}\right)^{2}\left(p_{11}+p_{22}-1\right)<a_{1}^{2}+a_{2}^{2}-a_{1}^{2} a_{2}^{2} .
$$

Thus, it is enough to set $a_{1}^{2}+a_{2}^{2}-a_{1}^{2} a_{2}^{2} \leqslant-1$ (for example, $a_{1}=\sqrt{2}$ and $a_{2}=\sqrt{3}$ ), to verify (19). In this situation, the recursive equation (17) does not converge to the steadystate solution, i.e., the system $\mathcal{S}$ is not stochastically stable, but it is $\tau$-stable. 


\section{Equivalence of stochastic $\tau$-stability concepts}

In this section a result concerning the equivalence among stochastic $\tau$-stability concepts is proven. First, the equivalence is proven in Lemma 6 assuming that $p_{i i}>0$ for each $i \in \mathfrak{X}$, and the complete result appears in Theorem 7. Consider

$$
X_{i}^{n}=E\left[z_{n} z_{n}^{\prime} \mathbb{1}_{\left\{\phi_{n}=i\right\}}\right], \quad \forall i \in \mathfrak{X},
$$

where $z_{n}=x_{T_{n}}$ and $\phi_{n}=\theta_{T_{n}}$, for $n=1, \ldots, N$.

Lemma 5. Suppose that $\mathcal{S}$ is $\tau$-SS. An equivalent form of expressing $X_{j}^{n} \in \mathcal{M}^{r 0}$ for $j \in \mathfrak{X}$ is given by

$$
X_{j}^{n}=\sum_{i \neq j} p_{i j} W_{i}^{n-1},
$$

where the matrices $W_{i}^{n} \in \mathcal{M}^{r}$ are obtained as the solution of the recursive matrix equation

$$
W_{i}^{k-1}=p_{i i} A_{i} W_{i}^{k-1} A_{i}^{\prime}+A_{i} X_{i}^{k-1} A_{i}^{\prime}, \quad k=1, \ldots, n-1,
$$

for each $i \in \mathfrak{X}$. Moreover,

$$
E\left[\left\|z_{n}\right\|^{2}\right]=\sum_{j \in \mathfrak{X}} \operatorname{tr}\left\{X_{j}^{n}\right\} \leqslant\left(\frac{\gamma}{1-\alpha^{2}}\right)^{n}\left\|x_{0}\right\|^{2},
$$

where $\alpha:=\max \left\{\lambda_{\max }\left(p_{i i}^{1 / 2} A_{i}\right)\right\}$ and $\gamma:=\max \left\{\left\|A_{i}\right\|^{2}\right\}$.

For the proof see Appendix A.

The following inequality will be useful. Set $c>0$; then for any $d_{0} \geqslant-1 /\left(e c^{4} \ln c\right)>0$ one has that

$$
k c^{2(k-2)} \leqslant d_{0} c^{k}, \quad \forall k \geqslant 0 .
$$

Lemma 6. For systems $\mathcal{S}$, where $0<p_{i i} \leqslant 1$ for all $i \in \mathfrak{X}, \tau-S S, \tau$-MSS and $\tau$-EMSS are equivalent.

Proof. Comparing (3), (4) and (5) notice that the implications $\tau$-SS $\Rightarrow \tau$-MSS and $\tau$-EMSS $\Rightarrow \tau$-MSS are immediately valid. It remains to show that $\tau$-SS $\Rightarrow \tau$-EMSS and $\tau$-MSS $\Rightarrow \tau$-SS.

$\tau$-SS $\Rightarrow \tau$-EMSS: The proof is obtained by an induction argument. Let $\hat{A}_{i}:=p_{i i}^{1 / 2} A_{i}$. Firstly consider $\tau=T_{1}$. In this case, it can be shown that

$$
E_{0}\left[\left\|x_{k}\right\|^{2} \mathbb{1}_{\left\{T_{1} \geqslant k\right\}}\right]=\left\|\hat{A}_{i}^{k-1} x_{0}\right\|^{2}\left\|A_{i}\right\|^{2} .
$$

Indeed, from the Lemma 2 we get that $P\left(T_{1}=m \mid \theta_{0}=i\right)=p_{i i}^{m-1}\left(1-p_{i i}\right) \mathbb{1}_{\left\{p_{i i}<1\right\}}$. Then

$$
\begin{aligned}
E_{0}\left[\left\|x_{k}\right\|^{2} \mathbb{1}_{\left\{T_{1} \geqslant k\right\}}\right] & =E_{0}\left[\left\|A_{i}^{k} x_{0}\right\|^{2} \mathbb{1}_{\left\{T_{1} \geqslant k\right\}}\right]=\sum_{m=k}^{+\infty}\left\|A_{i}^{k} x_{0}\right\|^{2} P\left(T_{1}=m \mid \theta_{0}=i\right) \\
& =\left\|\hat{A}_{i}^{k-1} x_{0}\right\|^{2}\left\|A_{i}\right\|^{2} \mathbb{1}_{\left\{p_{i i}<1\right\}} .
\end{aligned}
$$


When $p_{i i}=1$, since $P\left(T_{1} \geqslant k \mid \theta_{0}=i\right)=1$ for any $k>0$, one has that $E_{0}\left[\left\|x_{k}\right\|^{2} \mathbb{1}_{\left\{T_{1} \geqslant k\right\}}\right]=$ $\left\|A_{i}^{k} x_{0}\right\|^{2}$ and (24) remains valid. From (24) and Theorem 3(iii), we get that

$$
\begin{aligned}
E_{0}\left[\left\|x_{k}\right\|^{2} \mathbb{1}_{\left\{T_{1} \geqslant k\right\}}\right] & =\left\|\hat{A}_{i}^{k-1} x_{0}\right\|^{2}\left\|A_{i}\right\|^{2} \\
& \leqslant \lambda_{\max }^{2(k-1)}\left(\hat{A}_{i}\right)\left\|A_{i}\right\|^{2}\left\|x_{0}\right\|^{2} \leqslant \lambda_{\max }^{k-1}\left(\hat{A}_{i}\right)\left\|A_{i}\right\|^{2}\left\|x_{0}\right\|^{2} .
\end{aligned}
$$

Recalling that $\alpha=\max \left\{\lambda_{\max }\left(\hat{A}_{i}\right)\right\}$ and $\gamma=\max \left\{\left\|A_{i}\right\|^{2}\right\}$, and setting $c_{1}=\alpha^{-1}$, we can write

$$
E\left[\left\|x_{k}\right\|^{2} \mathbb{1}_{\left\{T_{1} \geqslant k\right\}}\right] \leqslant \gamma \alpha^{k-1}\left\|x_{0}\right\|^{2}=c_{1} \gamma \alpha^{k}\left\|x_{0}\right\|^{2} .
$$

Adopting $\beta=c_{1} \gamma>0$ we have that (5) holds for $\tau=T_{1}$.

Now, for the case $\tau=T_{n+2}$, first note that

$$
E\left[\left\|x_{k}\right\|^{2} \mathbb{1}_{\left\{T_{n+2} \geqslant k\right\}}\right]=E\left[\left\|x_{k}\right\|^{2} \mathbb{1}_{\left\{T_{n+1} \geqslant k\right\}}\right]+E\left[\left\|x_{k}\right\|^{2} \mathbb{1}_{\left\{T_{n+1}<k \leqslant T_{n+2}\right\}}\right] .
$$

Considering the induction hypothesis (see (25)), there exists a constant $c_{n+1}>0$ such that

$$
E\left[\left\|x_{k}\right\|^{2} \mathbb{1}_{\left\{T_{n+1} \geqslant k\right\}}\right] \leqslant c_{n+1} \gamma^{n+1} \alpha^{k}\left\|x_{0}\right\|^{2} .
$$

Regarding the second term in the right-hand side of (26), applying the strong Markov property and the homogeneity property (see (24)), it can be written as

$$
\begin{aligned}
& E\left[\left\|x_{k}\right\|^{2} \mathbb{1}_{\left\{T_{n+1}<k \leqslant T_{n+2}\right\}}\right]=E\left[E_{T_{n+1}}\left[\left\|x_{k}\right\|^{2} \mathbb{1}_{\left\{T_{n+1}<k \leqslant T_{n+2}\right\}}\right]\right] \\
& \quad=E\left[\left\|\hat{A}_{\phi_{n+1}-T_{n+1}-1} z_{n+1}\right\|^{2}\left\|A_{\phi_{n+1}}\right\|^{2} \mathbb{1}_{\left\{T_{n+1}<k\right\}}\right] \\
& \quad=E\left[\sum_{m=1}^{k-1} \sum_{j \in \mathfrak{X}} E_{T_{n}}\left[\left\|\hat{A}_{j}^{k-m-1} A_{\phi_{n}}^{m-T_{n}} z_{n}\right\|^{2}\left\|A_{j}\right\|^{2} \mathbb{1}_{\left\{\theta_{m}=j, T_{n+1}=m\right\}}\right]\right] .
\end{aligned}
$$

Now, applying Lemma 2 for $0<p_{\phi_{n} \phi_{n}}<1$, (28) is equivalent to

$$
\begin{aligned}
& E\left[\sum_{m=1}^{k-1} \sum_{j \neq \phi_{n}}\left\|\hat{A}_{j}^{k-m-1} A_{\phi_{n}}^{m-T_{n}} z_{n}\right\|^{2}\left\|A_{j}\right\|^{2} p_{\phi_{n} \phi_{n}}^{m-1-T_{n}} p_{\phi_{n} j} \mathbb{1}_{\left\{p_{\phi_{n} \phi_{n}}<1\right\}}\right] \\
& =\sum_{m=1}^{k-1} E\left[\sum_{j \neq \phi_{n}}\left\|\hat{A}_{j}^{k-m-1}\right\|^{2}\left\|\hat{A}_{\phi_{n}}^{m-T_{n}-1} z_{n}\right\|^{2}\left\|A_{j}\right\|^{2}\left\|A_{\phi_{n}}\right\|^{2} p_{\phi_{n} j} \mathbb{1}_{\left\{p_{\phi_{n} \phi_{n}}<1\right\}}\right] .
\end{aligned}
$$

Using the fact that $0<E\left[\alpha^{-2 T_{n}}\right] \leqslant E\left[\alpha^{-2 T_{N}}\right]:=\rho$ and considering the inequality (23) for $c=\alpha$, from (28) and (29) one has that

$$
\begin{aligned}
& E\left[E_{T_{n+1}}\left[\left\|x_{k}\right\|^{2} \mathbb{1}_{\left\{T_{n+1}<k \leqslant T_{n+2}\right\}}\right]\right] \\
& \quad \leqslant \sum_{m=1}^{k-1} E\left[\sum_{j \neq \phi_{n}} \lambda_{\max }^{2(k-m-1)}\left(\hat{A}_{j}\right) \lambda_{\max }^{2\left(m-T_{n}-1\right)}\left(\hat{A}_{\phi_{n}}\right)\left\|z_{n}\right\|^{2}\left\|A_{j}\right\|^{2}\left\|A_{\phi_{n}}\right\|^{2} p_{\phi_{n} j}\right] \\
& \quad \leqslant \sum_{m=1}^{k-1} \alpha^{2(k-2)} \gamma^{2} E\left[\sum_{j \neq \phi_{n}} \alpha^{-2 T_{n}}\left\|z_{n}\right\|^{2} p_{\phi_{n} j}\right] \\
& \quad \leqslant k \alpha^{2(k-2)} \gamma^{2} E\left[\alpha^{-2 T_{N}}\right] E\left[\left\|z_{n}\right\|^{2}\right] \leqslant d_{0} \rho \gamma^{2} \alpha^{k} E\left[\left\|z_{n}\right\|^{2}\right] .
\end{aligned}
$$


By setting $d_{1}=\left(1 / 1-\alpha^{2}\right)^{n}>0$ and applying (22), one has that

$$
E\left[E_{T_{n+1}}\left[\left\|x_{k}\right\|^{2} \mathbb{1}_{\left\{T_{n+1}<k \leqslant T_{n+2}\right\}}\right]\right] \leqslant d_{0} d_{1} \rho \gamma^{n+2} \alpha^{k}\left\|x_{0}\right\|^{2} .
$$

Finally, substituting (27) and (30) into (26) and setting $c_{n+2}=c_{n+1} \gamma^{-1}+d_{0} d_{1} \rho$, it follows that

$$
E\left[\left\|x_{k}\right\|^{2} \mathbb{1}_{\left\{T_{n+2} \geqslant k\right\}}\right] \leqslant c_{n+2} \gamma^{n+2} \alpha^{k}\left\|x_{0}\right\|^{2}, \quad \forall k>0 \text { and } 0 \leqslant n \leqslant N-2 .
$$

Therefore, by adopting $\beta=c_{n+2} \gamma^{n+2}>0$, (5) is valid for $\tau=T_{n+2}$. On the other hand, whenever $p_{\phi_{n} \phi_{n}}=1$, the second term in the right-hand side of (26) is null, since $P\left(T_{n+1}=\right.$ $\left.+\infty \mid \theta_{T_{n}}\right)=1$, and consequently the result follows straightforward from (27) with $\beta=$ $c_{n+1} \gamma^{n+1}>0$, completing this part of proof.

$\tau$-MSS $\Rightarrow \tau$-SS: Consider $\tau=T_{N}$. We can write

$$
E\left[\left\|x_{k}\right\|^{2} \mathbb{1}_{\left\{T_{N} \geqslant k\right\}}\right]=E\left[\left\|x_{k}\right\|^{2} \mathbb{1}_{\left\{T_{1} \geqslant k\right\}}\right]+E\left[\left\|x_{k}\right\|^{2} \mathbb{1}_{\left\{T_{1}<k \leqslant T_{N}\right\}}\right] .
$$

Assuming that $\lim _{k \rightarrow \infty} E\left[\left\|x_{k}\right\|^{2} \mathbb{1}_{\left\{T_{N} \geqslant k\right\}}\right]=0$, one has that $\lim _{k \rightarrow \infty} E\left[\left\|x_{k}\right\|^{2} \mathbb{1}_{\left\{T_{1} \geqslant k\right\}}\right]=0$. It follows from (24) that $r_{\sigma}\left(p_{i i}^{1 / 2} A_{i}\right)<1, \forall i \in \mathfrak{X}$. This implies that $\mathcal{S}$ is $\tau$-SS by Theorem 3(iii).

Theorem 7. For systems $\mathcal{S}, \tau-S S, \tau-M S S$ and $\tau$-EMSS are equivalent.

Proof. In view of Lemma 6, it remains to prove the case $p_{i i}=0$ for some $i \in \mathfrak{X}$.

$\tau$-SS $\Rightarrow \tau$-EMSS: Consider $\tau=T_{1}$. If $p_{i i}=0$, then $P\left(T_{1}=1 \mid \theta_{0}=i\right)=1$ and

$$
E_{0}\left[\left\|x_{k}\right\|^{2} \mathbb{1}_{\left\{T_{1} \geqslant k\right\}}\right]= \begin{cases}\left\|x_{0}\right\|^{2} & \text { if } k=0, \\ \left\|A_{i} x_{0}\right\|^{2} & \text { if } k=1, \\ 0 & \text { if } k>1\end{cases}
$$

Consequently,

$$
E_{0}\left[\left\|x_{k}\right\|^{2} \mathbb{1}_{\left\{T_{1} \geqslant k\right\}}\right] \leqslant \alpha^{k-1} \gamma\left\|x_{0}\right\|^{2}
$$

holds for all $k \geqslant 0$ and arbitrary $0<\alpha<1$. Assuming that $\beta=\gamma \alpha^{-1}$, (5) becomes true.

Now, for $\tau=T_{n+2}$, let us suppose that $p_{\phi_{n+1} \phi_{n+1}}=0$. This implies $P\left(T_{n+2}=\right.$ $\left.T_{n+1}+1\right)=1$, and applying both the strong Markov property and the homogeneity property (see (33)) one has that

$$
\begin{aligned}
E\left[\left\|x_{k}\right\|^{2} \mathbb{1}_{\left\{T_{n+1}<k \leqslant T_{n+2}\right\}}\right] & =E\left[E_{T_{n+1}}\left[\left\|x_{k}\right\|^{2} \mathbb{1}_{\left\{T_{n+1}<k \leqslant T_{n+1}+1\right\}}\right]\right] \\
& \leqslant E\left[\alpha^{k-1} \gamma\left\|z_{n+1}\right\|^{2}\right] .
\end{aligned}
$$

Here, by setting $d_{1}=\left(1 / 1-\alpha^{2}\right)^{n+1}>0$ and applying (22), we can write

$$
E\left[\left\|x_{k}\right\|^{2} \mathbb{1}_{\left\{T_{n+1}<k \leqslant T_{n+2}\right\}}\right] \leqslant \alpha^{k-1} d_{1} \gamma^{n+2}\left\|x_{0}\right\|^{2} .
$$

By substituting (27) and (34) into (26) and setting $c_{n+2}=c_{n+1} \gamma^{-1}+d_{1} \alpha^{-1}$, (31) holds. Hence, the proof is completed by adopting $\beta=c_{n+2} \gamma^{n+2}>0$.

$\tau$-MSS $\Rightarrow \tau$-SS: Consider $\tau=T_{1}$ and suppose that $p_{i i}=0$. From (32) notice that $E\left[\sum_{k \geqslant 0}\left\|x_{k}\right\|^{2} \mathbb{1}_{\left\{T_{1} \geqslant k\right\}}\right]<\infty$ and the $\tau$-SS is obtained by definition (see (3)). 
For the case $\tau=T_{n+2}$, consider $p_{\phi_{n+1} \phi_{n+1}}=0$. Using the induction hypothesis, suppose that $\lim _{k \rightarrow \infty} E\left[\left\|x_{k}\right\|^{2} \mathbb{1}_{\left\{T_{n+1} \geqslant k\right\}}\right]=0$ implies $E\left[\sum_{k \geqslant 0}\left\|x_{k}\right\|^{2} \mathbb{1}_{\left\{T_{n+1} \geqslant k\right\}}\right]<\infty$. Suppose also that $\lim _{k \rightarrow \infty} E\left[\left\|x_{k}\right\|^{2} \mathbb{1}_{\left\{T_{n+2} \geqslant k\right\}}\right]=0$. We wish to show that

$$
E\left[\sum_{k \geqslant 0}\left\|x_{k}\right\|^{2} \mathbb{1}_{\left\{T_{n+2} \geqslant k\right\}}\right]<\infty
$$

holds, since by (3) this fact ensure the $\tau$-SS. However, using (26) it remains to show that

$$
E\left[\sum_{k \geqslant 0}\left\|x_{k}\right\|^{2} \mathbb{1}_{\left\{T_{n+1}<k \leqslant T_{n+2}\right\}}\right]<\infty .
$$

But, this result is immediate from the fact that

$$
E_{0}\left[\left\|x_{k}\right\|^{2} \mathbb{1}_{\left\{T_{n+1}<k \leqslant T_{n+1}+1\right\}}\right]= \begin{cases}0 & \text { if } k \neq T_{n+1}+1 \\ \left\|A_{\phi_{n+1}} z_{n+1}\right\|^{2} & \text { if } k=T_{n+1}+1\end{cases}
$$

It follows the equivalence among $\tau$-SS, $\tau$-MSS and $\tau$-EMSS as claimed.

\section{Almost sure $\tau$-stability}

The almost sure (or sample path) stability is a well suited property for real systems, because the sample path behaviour can be observed in practice. Contrary to deterministic systems, for which all moments are stable whenever the sample path is stable, the moment stability for stochastic systems implies almost sure stability, but not vice versa, as pointed out by Kozin [7]. If we consider that moments can often be obtained or approximated, it is of interest to find out any implications in moment properties that leads to almost sure stability.

In the last years, many results concerning the almost sure stability are to be found in the literature. Regarding systems (1) and (2), Ji et al. [2] have shown that second moment stability implies almost sure stability, but the converse is not true. They have established that almost sure stability of the system is determined by the almost sure stability of its subsystems. The development of the Lyapunov exponent method provided a tool for the study of almost sure stability of stochastic systems, which leads to necessary and sufficient conditions, as demonstrated by Ji et al. [2] and Fang et al. [8].

In this section the almost sure $\tau$-stability concept is introduced and Theorem 9 states that second moment $\tau$-stability implies almost sure $\tau$-stability.

Definition 8. The MJLS $\mathcal{S}$ is almost surely $\tau$-stable, if for every initial state $x_{0}$ and initial distribution $\mu$,

$$
P\left(\lim _{k \rightarrow \infty}\left\|x_{k}\left(x_{0}, \theta_{0}\right)\right\| \mathbb{1}_{\{\tau \geqslant k\}}=0\right)=1 .
$$

Theorem 9. For the MJLS S, second moment $\tau$-stability implies almost sure $\tau$-stability. 
Proof. Suppose that systems is $\tau$-SS, i.e., $\sum_{k=0}^{\infty} E\left[\left\|x_{k}\right\|^{2} \mathbb{1}_{\{\tau \geqslant k\}}\right] \leqslant \infty$. From Chebishev's inequality,

$$
\sum_{k=0}^{\infty} P\left(\left\|x_{k}\right\|^{2} \mathbb{1}_{\{\tau \geqslant k\}} \geqslant \epsilon\right) \leqslant \frac{1}{\epsilon^{2}} \sum_{k=0}^{\infty} E\left[\left\|x_{k}\right\|^{2} \mathbb{1}_{\{\tau \geqslant k\}}\right] \leqslant \infty .
$$

Then from the first Borel-Cantelli lemma, it follows that

$$
P\left(\limsup _{k \rightarrow \infty}\left\|x_{k}\right\|^{2} \mathbb{1}_{\{\tau \geqslant k\}} \geqslant \epsilon\right)=0,
$$

and thus, $P\left(\lim _{k \rightarrow \infty}\left\|x_{k}\right\| \mathbb{1}_{\{\tau \geqslant k\}}=0\right)=1$.

\section{Conclusion}

The stochastic stability of discrete-time Markovian jump linear systems limited to the occurrence of a random event $\tau$ is studied here. The event represents the occurrence of a fixed number of failures, after which the system is paralyzed. Although in the literature there exists stochastic stability concepts for MJLS systems in general, previous notions show to be excessively conservative. In this sense the concept of stochastic $\tau$-stability developed here is more adequate to the deal with the stability in the formulated problem. We demonstrate that the concept is less conservative, and it is associated with a condition that is necessary but not sufficient for stochastic stability in the standard infinite horizon scenario as noted by Ji and Chizeck [1]. The related concept of almost sure stability is also studied here.

\section{Appendix A. Proof of Lemma 5}

The result is shown by induction on $n$. Let $n=1$.

Case $p_{i i}>0$ : Using Lemma 2, it follows that

$$
X_{j}^{1}=\sum_{i \in \mathfrak{X}} \sum_{k \geqslant 1} E\left[x_{k} x_{k}^{\prime} \mathbb{1}_{\left\{\theta_{k}=j, T_{1}=k, \theta_{0}=i\right\}}\right]=\sum_{i \in \mathfrak{X}} \sum_{k \geqslant 1} A_{i}^{k} x_{0} x_{0}^{\prime} A_{i}^{\prime k} p_{i i}^{k-1} p_{i j} \mathbb{1}_{\left\{p_{i i}<1\right\}} .
$$

Identifying $X_{i}^{0}$, the last expression is equivalent to

$$
X_{j}^{1}=\sum_{i \in \mathfrak{X}}\left(\sum_{k \geqslant 1} A_{i}^{k} X_{i}^{0} A_{i}^{\prime k} p_{i i}^{k-1} p_{i j}\right)=\sum_{i \in \mathfrak{X}} p_{i j} W_{i}^{0}
$$

where $W_{i}^{0}=\sum_{k \geqslant 1} A_{i}^{k} X_{i}^{0} A_{i}^{\prime k} p_{i i}^{k-1}$.

Applying the induction hypothesis, with the fact that the system is $\tau$-SS, one gets that

$$
W_{i}^{0}=A_{i} X_{i}^{0} A_{i}^{\prime}+\sum_{k \geqslant 2} A_{i}^{k} X_{i}^{0} A_{i}^{\prime k} p_{i i}^{k-1}=A_{i} X_{i}^{0} A_{i}^{\prime}+p_{i i} A_{i}\left(\sum_{k \geqslant 1} A_{i}^{k} X_{i}^{0} A_{i}^{\prime k} p_{i i}^{k-1}\right) A_{i}^{\prime} .
$$

Notice that the matrix $W_{i}^{0}$ can be recognized between the parenthesis above, and Eq. (21) is thus verified. 
Case $p_{i i}=0$ : Equivalently, using Lemma 2 , since $P\left(T_{1}=1 \mid \theta_{0}=1\right)=1$,

$$
X_{j}^{1}=\sum_{i \in \mathfrak{X}} A_{i} x_{0} x_{0}^{\prime} A_{i}^{\prime} p_{i j} .
$$

In this situation, it is enough to set $W_{i}^{0}=A_{i} X_{i}^{0} A_{i}^{\prime}$. For $n>1$, it is enough to use the strong Markov property to conclude that (21) holds. For the second part of lemma, observe that

$$
E\left[\left\|z_{n}\right\|^{2}\right]=\operatorname{tr}\left\{\sum_{j \in \mathfrak{X}} X_{j}^{n}\right\}=\sum_{i \in \mathfrak{X}} \operatorname{tr}\left\{W_{i}^{n-1}\right\}\left(1-p_{i i}\right) .
$$

Also,

$$
W_{i}^{n-1}=\sum_{k \geqslant 1} A_{i}^{k} X_{i}^{n-1} A_{i}^{\prime k} p_{i i}^{k-1} \leqslant \sum_{k \geqslant 1} \lambda_{\max }^{2(k-1)}\left(p_{i i} A_{i}\right) A_{i}^{\prime} X_{i}^{n-1} A_{i} .
$$

Setting $\alpha$ and $\gamma$ as in the Lemma, (A.1) yields that

$$
E\left[\left\|z_{n}\right\|^{2}\right] \leqslant\left(\frac{\gamma}{1-\alpha^{2}}\right) \sum_{i \in \mathfrak{X}} \operatorname{tr}\left\{X_{i}^{n-1}\right\} \leqslant\left(\frac{\gamma}{1-\alpha^{2}}\right) E\left[\left\|z_{n-1}\right\|^{2}\right] .
$$

Finally, the inequality (22) is obtained by iteration of the above relation.

\section{References}

[1] Y. Ji, H.J. Chizeck, Jump linear quadratic Gaussian control: Steady-state solution and testable conditions, Control Theory Adv. Technol. 6 (1990) 289-319.

[2] Y. Ji, H.J. Chizeck, K.A. Loparo, Stability and control of discrete-time jump linear systems, Control Theory Adv. Technol. 7 (1991) 247-270.

[3] O.L.V. Costa, M.D. Fragoso, Stability results for discrete-time linear systems with Markovian jumping parameters, J. Math. Anal. Appl. 179 (1993) 154-178.

[4] Y. Fang, A new general sufficient condition for almost sure stability of jump linear systems, IEEE Trans. Automat. Control 42 (1997) 378-382.

[5] Z.G. Li, Y.C. Soh, C.Y. Wen, Sufficient conditions for almost sure stability of jump linear systems, IEEE Trans. Automat. Control 45 (2000) 1325-1329.

[6] K. Zhou, J.C. Doyle, K. Glover, Robust and Optimal Control, Prentice-Hall, 1996.

[7] F. Kozin, A survey of stability of stochastic systems, Automatica 5 (1969) 95-112.

[8] Y. Fang, K.A. Loparo, X. Feng, Almost sure and $\delta$-moment stability of jump linear systems, Internat. J. Control 59 (1994) 1281-1307. 